\title{
Detailed Mathematical Analysis of Harmonics in Multi-Phase Inverters for Hybrid Electric Vehicles
}

\author{
Sangeeta Sahu*, Byamakesh Nayak and Rudra Narayan Dash
}

KIIT, Bhubaneswar, Odisha, 751024, India

Received 3 December 2019; Accepted 6 November 2020

\begin{abstract}
This paper presents a comparative analysis of power handling capability, harmonics, losses and cost of three phase, five phase and seven phase inverter which are the main criteria for selection of inverter applied to hybrid electric vehicle. The above performances of the multiphase inverter with different conduction angle are presented to trade off the best suited inverter for hybrid electric vehicle application. The $6^{\text {th }}$ harmonic pulsating torque created due to $5^{\text {th }}$ and $7^{\text {th }}$ harmonic component and $12^{\text {th }}$ harmonic pulsating torque created due to $11^{\text {th }}$ and $13^{\text {th }}$ harmonic which affect the smooth movement of the vehicle at the time of starting and braking condition. This paper gives a clear idea about the comparison of the above harmonic component for different conduction angle scenario of 3 phase, 5 phase and 7 phase inverter. The equations of the performance criteria such output voltage, THD, power handling, losses, etc has been mathematically estimated and verified through Matlab Simulink Power Graphical User Interface.
\end{abstract}

Keywords: Harmonics, Multi-Phase Inverter, Phase Voltage, THD, Hybrid Electric Vehicle

\section{Introduction}

An inverter circuit topology uses two switches connected in series as one inverter arm. The number of inverter arms depends on number of phases. That is, a three phase inverter will have three inverter arms whereas a seven phase inverter circuit will have seven inverter arms. All the inverter circuits used in literature for multiphase inverters employ the same topology. In multi phase inverters, the conduction angle can be varied to get optimum output. Depending on the application, the switches used in inverters can be IGBT, MOSFET, GTO, etc. Each of the switches have diodes connected across them and these diodes act as feedback diodes which returns the energy stored in the inductive load to the dc supply. A comparative study on the total harmonic distortion of the output phase voltage at different conduction angle has been presented in this paper.

With the decrease in harmonic profile, the ripple in the torque produced in electric vehicles is reduced. For 5th and 7 th harmonic, the torque ripple is pulsating with 6 times the fundamental frequency. For 11th and 13th harmonic, the torque ripple is pulsating at 12 times the fundamental frequency. Due to these harmonics, it produces noise, humming sound and jerking at the time of braking. For the same input voltage the power handling capability increases with the increase in the number of phases of the motor. But at the same time, the cost of inverter as well as of the control procedure increases with increase in number of phases. So, there must be a tradeoff between power handling capability, THD and cost of inverter. The $5^{\text {th }}$ and $7^{\text {th }}$ phase inverters can be used in hybrid electric vehicles. Multisource inverter can drive traction motors from variable DC voltages without

*E-mail address: Sahu.sangeeta@gmail.com

ISSN: 1791-2377 @ 2020 School of Science, IHU. All rights reserved. doi:10.25103/jestr.136.18 using additional power converters has application in electrified power trains [1]. The transient analysis of high power VSI shows that a VSI is always a stable system with nonlinearities and unstable with respect to control signal [2]. The THD and switching losses are reduced considerably a dual voltage source inverter which is implemented through a modified space vector modulation [3]. A six phase current reconstruction scheme for dual inverters that have application in hybrid electric vehicle is presented in [4]. In this method the simulation and experimental results proof that the cost and volume of dual inverters are reduced as less number of current sensors are used. A bidirectional $\mathrm{Z}$ - source inverter has been recommended for the hybrid electric vehicle drive system in which the DC-link voltage is controlled such that the permanent magnet synchronous motor can operate at high speed without weakening the field [5]. The magnetic field created by the inverter in electric vehicles is evaluated using finite element method [6] and few design guidelines have been provided to minimize the same.

The fast development of power electronic devices and modern control theory had made the study and applications of five phase permanent magnet synchronous motor as well as its driving system that is the voltage source inverters attract more and more attention. Five phase PMSM has wide applications requiring high DC bus voltage utilization, reliability and power output [7]. THD comparison for a three phase inverter at different conduction mode concludes that 150 deg conduction mode has least THD [8-9]. PWM operation of a five phase voltage source inverter with hysteresis controller can be applied to a variable speed induction motor drive under asymmetrical connections [10]. A comparative study of performance of five phase three level inverter with five phase two level inverter for equal loading shows that five phase three level gives better performance [11]. A five phase inverter fed five phase star connected load operating with five different excitations has been simulated 
and compared with that of three phase conventional inverter [12]. The ripple in torque and harmonics can be reduced by supply a motor with multi phase supply that is greater than three phase supply [13]. A multiphase inverter with phaseshifted control is applied in electric vehicles for inductive power transfer [14]. Here the inverter phase angle is adjusted to regulate the charging voltage. The implementation of space vector pulse width modulation for five phase inverter is very simplifies the computationally complexity [15-16]. Current imbalance between stator windings that results in shortening of the life of motor can be eliminated by symmetrical multiphase inverters [17].

\section{Structure of a Multiphase Inverter}

The multiphase inverter consists of multiple legs with two switches in each leg and the number of legs equals to the number of phases. Each switch has a diode connected in antiparallel with it. The gating pulse in two adjacent switches has a delay of $(360 / \mathrm{m})^{0}$ where $n$ is the number of phases. The gate pulse between two switches of same leg has a delay of $180^{\circ}$. The inverter is connected to a star connected resistive load. The schematic diagram of seven phase full bridge voltage source inverter is shown in Fig.1.

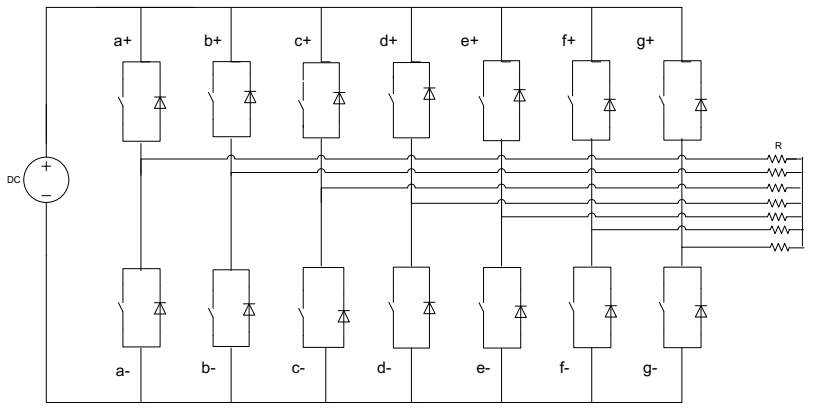

Fig. 1. Schematic diagram of Seven Phase Inverter

\section{Conduction Techniques in Multiphase Inverters}

In industries, variable frequency and voltages are required for various applications. The switching of the semiconductor switches in an inverter depends on the firing angle giving to the switches. Multiphase inverters are operated in different conduction modes. There are different modes on conduction in the multiphase inverters that decides the period for which the switches shall conduct. The conduction angle of a switch is given by the formula

$$
\left(180-\frac{360}{2 m} P\right)
$$

where $\mathrm{P}$ may vary from 0 to $(\mathrm{m}-1)$ and $\mathrm{m}$ is the number of phases.

For $\mathrm{P}=0$, that is $180 \mathrm{deg}$ mode of conduction, each switch conducts for $180 \mathrm{deg}$. At any particular instant, all the switches are in $\mathrm{ON}$ state. Similarly for $\mathrm{P}=1,(\mathrm{~m}-1)$ switches are in ON state at any instant. For $\mathrm{P}=1 / 2$, the switching sequence changes alternatively between $\mathrm{n}$ and $(\mathrm{m}-1)$ switches for each $(360 / 4 \mathrm{~m})$ deg. Tables 1,2 and 3 shows the switching sequence for $\mathrm{P}=0,1$ and $1 / 2$ for three phase, five phase and seven phase inverters respectively.

\section{Output Voltage Waveform of Multiphase Inverters}

The phase and line voltage waveform for 3,5 and 7 phase inverters at $\mathrm{P}=0,1 / 2$ and 1 is shown in Figs. 2 and 3 respectively. At $\mathrm{P}=1 / 2$ conduction angle, the inverter can be considered as a seven level inverter as the phase voltage output has seven levels. The significant advantage of this output waveform is that it is close to sinusoidal. Since higher the number of levels, closer is the waveform to sinusoidal wave. This in turn reduces the stress on the motor's insulation level.

Table 1. Switching sequence for $P=0$

\begin{tabular}{|c|c|c|c|c|c|c|c|}
\hline $\begin{array}{c}\text { Switching } \\
\text { Mode }\end{array}$ & 3 Phase & 5 Phase & 7 Phase & $\begin{array}{c}\text { Switching } \\
\text { Mode }\end{array}$ & 3 Phase & 5 Phase & 7 Phase \\
\hline 1 & $\begin{array}{c}a^{+} c^{+} \\
b^{-}\end{array}$ & $\begin{array}{c}a^{+} d^{+} e^{+} \\
b^{-} c^{-}\end{array}$ & $\begin{array}{c}a^{+} e^{+} f^{+} g^{+} \\
b^{-} c^{-} d^{-}\end{array}$ & 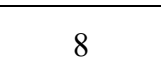 & - & $\begin{array}{c}c^{+} d^{+} \\
a^{-} b^{-} e^{-}\end{array}$ & $\begin{array}{c}\mathrm{b}^{+} \mathrm{c}^{+} \mathrm{d}^{+} \\
\mathrm{a}^{-} \mathrm{e}^{-} \mathrm{f}^{-} \mathrm{g}^{-}\end{array}$ \\
\hline 2 & $\begin{array}{c}\mathrm{a}^{+} \\
\mathrm{b}^{-} \mathrm{c}^{-}\end{array}$ & $\begin{array}{c}\mathrm{a}^{+} \mathrm{e}^{+} \\
\mathrm{b}^{-} \mathrm{c}^{-} \mathrm{d}^{-}\end{array}$ & $\begin{array}{c}a^{+} f^{+} g^{+} \\
b^{-} c^{-} d^{-} e^{-}\end{array}$ & 9 & - & $\begin{array}{c}\mathrm{c}^{+} \mathrm{d}^{+} \mathrm{e}^{+} \\
\mathrm{a}^{-} \mathrm{b}^{-}\end{array}$ & $\begin{array}{c}\mathrm{b}^{+} \mathrm{c}^{+} \mathrm{d}^{+} \mathrm{e}^{+} \\
\mathrm{a}^{-} \mathrm{f}^{-} \mathrm{g}^{-}\end{array}$ \\
\hline 3 & $\begin{array}{c}\mathrm{a}^{+} \mathrm{b}^{+} \\
\mathrm{c}^{-}\end{array}$ & $\begin{array}{c}a^{+} b^{+} e^{+} \\
c^{-} d^{-}\end{array}$ & $\begin{array}{c}\mathrm{a}^{+} \mathrm{b}^{+} \mathrm{f}^{+} \mathrm{g}^{+} \\
\mathrm{c}^{-} \mathrm{d}^{-} \mathrm{e}^{-}\end{array}$ & 10 & - & $\begin{array}{c}d^{+} e^{+} \\
a^{-} b^{-} c^{-}\end{array}$ & $\begin{array}{c}c^{+} d^{+} e^{+} \\
a^{-} b^{-} f^{-} g^{-}\end{array}$ \\
\hline 4 & $\begin{array}{c}\mathrm{b}^{+} \\
\mathrm{a}^{-} \mathrm{c}^{-}\end{array}$ & $\begin{array}{c}a^{+} b^{+} \\
c^{-} d^{-} e^{-}\end{array}$ & $\begin{array}{l}a^{+} b^{+} g^{+} \\
c^{-} d^{-} e^{-} f^{-}\end{array}$ & 11 & - & 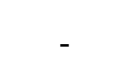 & $\begin{array}{c}c^{+} d^{+} e^{+} f^{+} \\
a^{-} b^{-} g^{-}\end{array}$ \\
\hline 5 & $\begin{array}{c}\mathrm{b}^{+} \mathrm{c}^{+} \\
\mathrm{a}^{-}\end{array}$ & $\begin{array}{c}a^{+} b^{+} c^{+} \\
d^{-} e^{-}\end{array}$ & $\begin{array}{c}a^{+} b^{+} c^{+} g^{+} \\
d^{-} e^{-} f^{-}\end{array}$ & 12 & - & - & $\begin{array}{c}d^{+} e^{+} f^{+} \\
a^{-} b^{-} c^{-} g^{-}\end{array}$ \\
\hline 6 & $\begin{array}{c}c^{+} \\
a^{-} b^{-}\end{array}$ & $\begin{array}{c}\mathrm{b}^{+} \mathrm{c}^{+} \\
\mathrm{a}^{-} \mathrm{d}^{-} \mathrm{e}^{-}\end{array}$ & $\begin{array}{c}a^{+} b^{+} c^{+} \\
d^{-} e^{-} f^{-} g^{-}\end{array}$ & 13 & - & - & $\begin{array}{c}\mathrm{d}^{+} \mathrm{e}^{+} \mathrm{f}^{+} \mathrm{g}^{+} \\
\mathrm{a}^{-} \mathrm{b}^{-} \mathrm{c}^{-}\end{array}$ \\
\hline 7 & - & $\begin{array}{c}\mathrm{b}^{+} \mathrm{c}^{+} \mathrm{d}^{+} \\
\mathrm{a}^{-} \mathrm{e}^{-}\end{array}$ & $\begin{array}{c}a^{+} b^{+} c^{+} d^{+} \\
e^{-} f^{-} g^{-}\end{array}$ & 14 & - & - & $\begin{array}{c}e^{+} f^{+} g^{+} \\
a^{-} b^{-} c^{-} d^{-}\end{array}$ \\
\hline
\end{tabular}

Table 2. Switching sequence for $P=1$

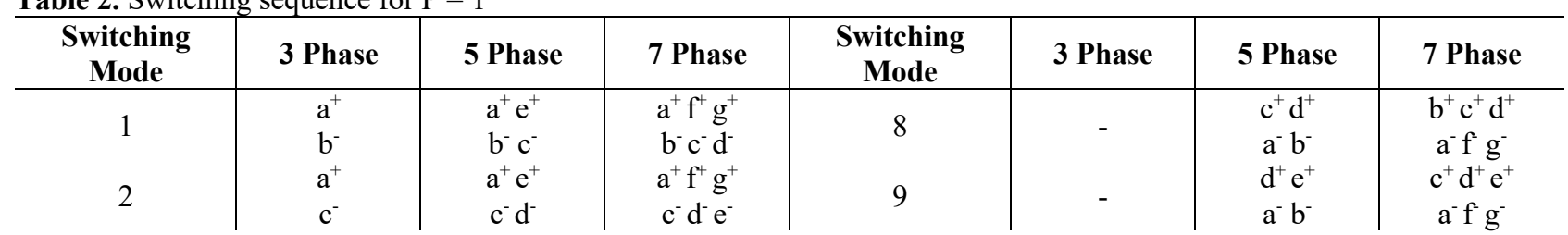


Sangeeta Sahu, Byamakesh Nayak and Rudra Narayan Dash/

Journal of Engineering Science and Technology Review 13 (6) (2020) 130 - 136

\begin{tabular}{|c|c|c|c|c|c|c|c|}
\hline & $\mathrm{b}^{+}$ & $\mathrm{a}^{+} \mathrm{b}^{+}$ & $a^{+} b^{+} g^{+}$ & & & $\mathrm{d}^{+} \mathrm{e}^{+}$ & $\mathrm{c}^{+} \mathrm{d}^{+} \mathrm{e}^{+}$ \\
\hline 3 & $\mathrm{c}^{-}$ & $\mathrm{c}^{-} \mathrm{d}^{-}$ & $\mathrm{c}^{-} \mathrm{d}^{-} \mathrm{e}^{-}$ & 10 & - & $b^{-} c^{-}$ & $a^{-} b^{-} g^{-}$ \\
\hline 4 & $\begin{array}{l}\mathrm{b}^{+} \\
\mathrm{a}^{-}\end{array}$ & $\begin{array}{l}\mathrm{a}^{+} \mathrm{b}^{+} \\
\mathrm{d}^{-} \mathrm{e}^{-}\end{array}$ & $\begin{array}{c}\mathrm{a}^{+} \mathrm{b}^{+} \mathrm{g}^{+} \\
\mathrm{d}^{-} \mathrm{e}^{-} \mathrm{f}^{-}\end{array}$ & 11 & - & - & $\begin{array}{l}d^{+} e^{+} f^{+} \\
a^{-} b^{-} g^{-}\end{array}$ \\
\hline 5 & $\begin{array}{l}\mathrm{c}^{+} \\
\mathrm{a}^{-}\end{array}$ & $\begin{array}{l}\mathrm{b}^{+} \mathrm{c}^{+} \\
\mathrm{d}^{-} \mathrm{e}^{-}\end{array}$ & $\begin{array}{c}a^{+} b^{+} c^{+} \\
d^{-} e^{-} f^{-}\end{array}$ & 12 & - & - & $\begin{array}{l}d^{+} e^{+} f^{+} \\
a^{-} b^{-} c^{-}\end{array}$ \\
\hline 6 & $\begin{array}{l}c^{+} \\
b^{-}\end{array}$ & $\begin{array}{l}\mathrm{b}^{+} \mathrm{c}^{+} \\
\mathrm{a}^{-} \mathrm{e}^{-}\end{array}$ & $\begin{array}{c}a^{+} b^{+} c^{+} \\
e^{-} f^{-} g^{-}\end{array}$ & 13 & - & - & $\begin{array}{l}\mathrm{e}^{+} \mathrm{f}^{+} \mathrm{g}^{+} \\
\mathrm{a}^{-} \mathrm{b}^{-} \mathrm{c}^{-}\end{array}$ \\
\hline 7 & - & $\begin{array}{l}\mathrm{c}^{+} \mathrm{d}^{+} \\
\mathrm{a}^{-} \mathrm{e}^{-}\end{array}$ & $\begin{array}{c}\mathrm{b}^{+} \mathrm{c}^{+} \mathrm{d}^{+} \\
\mathrm{e}^{-} \mathrm{f}^{-} \mathrm{g}^{-}\end{array}$ & 14 & - & - & $\begin{array}{l}\mathrm{e}^{+} \mathrm{f}^{+} \mathrm{g}^{+} \\
\mathrm{b}^{-} \mathrm{c}^{-} \mathrm{d}^{-}\end{array}$ \\
\hline
\end{tabular}

Table 3. Switching sequence for $\mathrm{P}=1 / 2$

\begin{tabular}{|c|c|c|c|c|c|c|c|}
\hline $\begin{array}{l}\text { Switching } \\
\text { Mode }\end{array}$ & 3 Phase & 5 Phase & 7 Phase & $\begin{array}{c}\text { Switching } \\
\text { Mode }\end{array}$ & 3 Phase & 5 Phase & 7 Phase \\
\hline (1) & $\begin{array}{l}a^{+} c^{+} \\
h^{-}\end{array}$ & $\begin{array}{l}a^{+} d^{+} e^{+} \\
b^{-} c^{-}\end{array}$ & $\begin{array}{l}a^{+} e^{+} f^{+} g^{+} \\
b^{-} c^{-} d^{-}\end{array}$ & 15 & - & $\begin{array}{c}c^{+} d^{+} \\
a^{-} b^{-} e^{-}\end{array}$ & $\begin{array}{l}b^{+} c^{+} d^{+} \\
a^{-} e^{-f} g^{-}\end{array}$ \\
\hline 2 & $\begin{array}{l}0 \\
\mathrm{a}^{+}\end{array}$ & $\mathrm{a}^{+} \mathrm{e}^{+}$ & $a^{+} f^{+} g^{+}$ & 16 & - & $\mathrm{c}^{+} \mathrm{d}^{+}$ & $b^{+} c^{+} d^{+}$ \\
\hline & $\begin{array}{l}\mathrm{b}^{-} \\
\mathrm{a}^{+}\end{array}$ & $\begin{array}{l}\mathrm{b}^{-} \mathrm{c}^{-} \\
\mathrm{a}^{+} \mathrm{e}^{+}\end{array}$ & $\begin{array}{c}b^{-} c^{-} d^{-} \\
a^{+} f^{+} g^{+}\end{array}$ & & & $\begin{array}{c}a^{-} b^{-} \\
c^{+} d^{+} e^{+}\end{array}$ & $\begin{array}{c}a^{a} f^{-g} \\
b^{+} c^{+} d^{+} e^{+}\end{array}$ \\
\hline 3 & $\mathrm{~b}^{\mathrm{a}} \mathrm{c}^{-}$ & $b^{-} c^{-} d^{-}$ & $b^{-} c^{-} d^{-} e^{-}$ & 17 & - & $a^{-} b^{-}$ & $a^{-} f^{-} g^{-}$ \\
\hline 4 & $\begin{array}{l}\mathrm{a}^{+} \\
\mathrm{c}^{-}\end{array}$ & $\begin{array}{l}\mathrm{a}^{+} \mathrm{e}^{+} \\
\mathrm{c}^{-} \mathrm{d}^{-}\end{array}$ & $\begin{array}{l}a^{+} f^{+} g^{+} \\
c^{-} d^{-} e^{-}\end{array}$ & 18 & - & $\begin{array}{l}d^{+} e^{+} \\
a^{-} b^{-}\end{array}$ & $\begin{array}{l}c^{+} d^{+} e^{+} \\
a^{-} f^{-} g^{-}\end{array}$ \\
\hline 5 & $\begin{array}{c}\mathrm{a}^{+} \mathrm{b}^{+} \\
\mathrm{c}^{-}\end{array}$ & $\begin{array}{c}\mathrm{a}^{+} \mathrm{b}^{+} \mathrm{e}^{+} \\
\mathrm{c}^{-} \mathrm{d}^{-}\end{array}$ & $\begin{array}{l}a^{+} b^{+} f^{+} g^{+} \\
c^{-} d^{-} e^{-}\end{array}$ & 19 & - & $\begin{array}{c}d^{+} e^{+} \\
a^{-} b^{-} c^{-}\end{array}$ & $\begin{array}{c}c^{+} d^{+} e^{+} \\
a^{-} b^{-} f^{-} g^{-}\end{array}$ \\
\hline 6 & $\begin{array}{l}\mathrm{b}^{+} \\
\mathrm{c}^{-}\end{array}$ & $\begin{array}{l}a^{+} b^{+} \\
c^{-} d^{-}\end{array}$ & $\begin{array}{l}\mathrm{a}^{+} \mathrm{b}^{+} \mathrm{g}^{+} \\
\mathrm{c}^{-} \mathrm{d}^{-} \mathrm{e}^{-}\end{array}$ & 20 & - & $\begin{array}{l}\mathrm{d}^{+} \mathrm{e}^{+} \\
\mathrm{b}^{-} \mathrm{c}^{-}\end{array}$ & $\begin{array}{l}c^{+} d^{+} e^{+} \\
a^{-} b^{-} g^{-}\end{array}$ \\
\hline 7 & $\begin{array}{c}\mathrm{b}^{+} \\
\mathrm{a}^{-} \mathrm{c}^{-}\end{array}$ & $\begin{array}{c}a^{+} b^{+} \\
c^{-} d^{-} e^{-}\end{array}$ & $\begin{array}{l}a^{+} b^{+} g^{+} \\
c^{-} d^{-} e^{-} f\end{array}$ & 21 & - & & $\begin{array}{c}c^{+} d^{+} e^{+} f^{+} \\
a^{-} b^{-} g^{-}\end{array}$ \\
\hline
\end{tabular}

$3 \mathrm{Ph}$

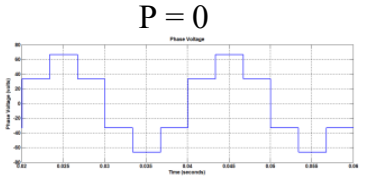

$5 \mathrm{Ph}$

$7 \mathrm{Ph}$
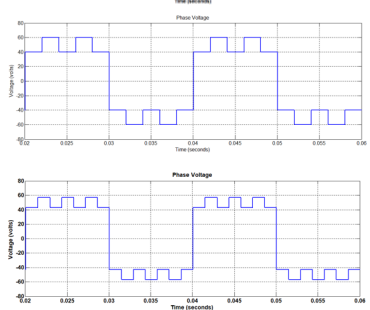

Fig. 2. Phase Voltage Waveform of Multiphase Inverters

$3 \mathrm{Ph}$

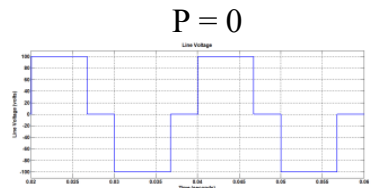

$5 \mathrm{Ph}$

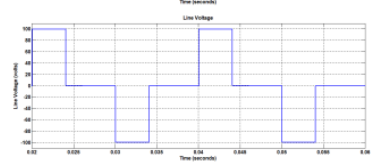

$7 \mathrm{Ph}$

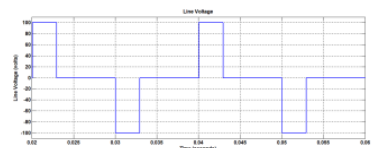

Fig. 3. Line Voltage Waveform of Multiphase Inverters

\section{Results and Discussion}

The phase voltage, line voltage, THD and loss comparison of three phase, five phase and seven phase inverters at $\mathrm{P}=0,1 / 2$
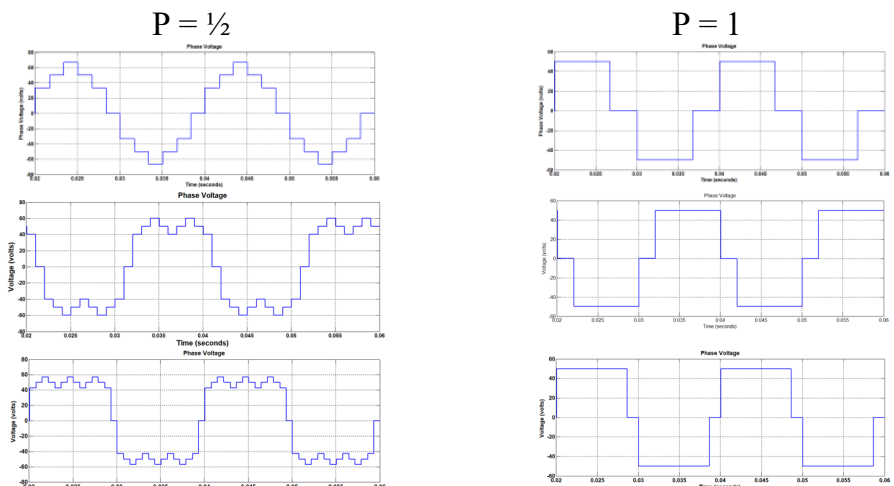
mentioned in the Appendix. The results are shown considering 1 p.u. input voltage. Here the switching loss, conduction loss and power handling capacity are considered as 1 p.u. for a three phase inverter. In a three phase inverter $P$ $=0$ conduction mode, three switches are in $\mathrm{ON}$ state at any instance. Thus conduction loss for three switches is considered as 1 . Similarly at every $60 \mathrm{deg}$ interval, one switch is changing its state. Thus switching loss for three phase inverter at $\mathrm{P}=0$ is considered as 1 . Considering three phase inverter at $\mathrm{P}=0$ as reference, the switching loss, conduction loss and power handling capacity of five phase and seven phase inverters are calculated.

The power handling capability of the 3 phase inverter is the lowest among higher phase inverter such as 5phase and 7 phase. In order to handle the same amount of power, the stresses on the switches increases in 3phase inverter this reduces the life span of the inverter. In addition, the $5^{\text {th }}, 7^{\text {th }}$, $11^{\text {th }}, 13^{\text {th }}$ harmonic components are more than the 5 phase and 7 phase inverter which creates $6^{\text {th }}$ and $12^{\text {th }}$ harmonic pulsating toque respectively that affect the machine performances. The
5 and 7 phase inverter may be considered for electric hybrid vehicle because of high power handling capability and less switching stress. The five phase inverter has no fifth harmonic and it's multiple whereas 7 phase inverter has no seventh harmonic and it's multiple. The 7 phase inverter has more $5^{\text {th }}$ harmonic component compared to $7^{\text {th }}$ harmonic component of 5 phase inverter. Therefore it is concluded that the $6^{\text {th }}$ harmonic pulsating torque created by 5 phase inverter is less compared to the 7 phase inverter. Besides, the $11^{\text {th }}$ and $13^{\text {th }}$ harmonic of 5 phase inverter is less as compared to the 7 phase inverter which reduces the $12^{\text {th }}$ harmonic pulsating torque in 5phase inverter. The FFT analysis of 5phase inverter at conduction angle $\mathrm{P}=1 / 2$ (i.e. $162 \mathrm{deg}$ ) is shown in Fig. 4. The FFT analysis of 3, 5 and 7 phase inverters at different conduction angle $\mathrm{P}$ is obtained in a similar manner. Table $\mathrm{V}$ shows the harmonic profile of 3,5 and 7 phase inverters and different conduction angle P. Figs. 5, 6 and 7 shows the graphical representation on multiphase inverters at $\mathrm{P}=0,1 / 2$ and 1 respectively.

Table 4. Comparison of three phase, five phase and seven phase inverter at different conduction modes considering 1 p.u. input voltage

\begin{tabular}{|c|c|c|c|c|c|c|c|c|c|c|c|}
\hline & Paramet & & $\begin{array}{c}\text { Phase } \\
\text { Voltage }\end{array}$ & $\begin{array}{c}\text { Line } \\
\text { Voltage }\end{array}$ & $\begin{array}{c}\text { THD in } \\
\text { Phase } \\
\text { Voltage }\end{array}$ & $\begin{array}{l}\text { THD in } \\
\text { Line } \\
\text { Voltage }\end{array}$ & $\mathrm{V}_{\text {0rms1 }}$ & $\mathbf{V}_{0 \mathrm{rms}}$ & $\begin{array}{l}\text { Switching } \\
\text { Loss }\end{array}$ & $\begin{array}{l}\text { Conduction } \\
\text { Loss }\end{array}$ & $\begin{array}{c}\text { Power } \\
\text { Handling }\end{array}$ \\
\hline \multirow{3}{*}{$\begin{array}{l}\text { Three } \\
\text { Phase } \\
\text { Inverter }\end{array}$} & $\mathrm{P}=0$ & $\begin{array}{c}\text { Sim. } \\
\text { Result } \\
\text { Cal. } \\
\text { Result } \\
\end{array}$ & $\begin{array}{l}0.639 \\
0.639\end{array}$ & $\begin{array}{l}1.10 \\
1.10\end{array}$ & $\begin{array}{c}30.9 \\
30.14\end{array}$ & $\begin{array}{l}30.9 \\
30.9\end{array}$ & $\begin{array}{l}0.45 \\
0.45\end{array}$ & $\begin{array}{c}- \\
0.47\end{array}$ & $\begin{array}{l}- \\
1\end{array}$ & $\begin{array}{l}- \\
1\end{array}$ & $\begin{array}{l}- \\
1\end{array}$ \\
\hline & $\mathrm{P}=1 / 2$ & $\begin{array}{c}\text { Sim. } \\
\text { Result } \\
\text { Cal. } \\
\text { Result }\end{array}$ & $\begin{array}{l}0.614 \\
0.614\end{array}$ & $\begin{array}{l}1.06 \\
1.06\end{array}$ & $\begin{array}{l}16.89 \\
16.89\end{array}$ & $\begin{array}{l}16.83 \\
16.83\end{array}$ & $\begin{array}{c}0.43 \\
0.434\end{array}$ & $\begin{array}{c}- \\
0.44\end{array}$ & $\begin{array}{l}- \\
2\end{array}$ & $\begin{array}{c}- \\
0.833\end{array}$ & $\begin{array}{c}- \\
0.923\end{array}$ \\
\hline & $\mathrm{P}=1$ & $\begin{array}{c}\text { Sim. } \\
\text { Result } \\
\text { Cal. } \\
\text { Result } \\
\end{array}$ & $\begin{array}{l}0.552 \\
0.552\end{array}$ & $\begin{array}{l}0.955 \\
0.955\end{array}$ & $\begin{array}{l}30.9 \\
31.6\end{array}$ & $\begin{array}{c}30.9 \\
31\end{array}$ & $\begin{array}{l}0.389 \\
0.389\end{array}$ & $\begin{array}{c}- \\
0.408\end{array}$ & $\begin{array}{l}- \\
2\end{array}$ & $\begin{array}{c}- \\
0.67\end{array}$ & $\begin{array}{c}- \\
0.746\end{array}$ \\
\hline \multirow{3}{*}{$\begin{array}{c}\text { Five } \\
\text { Phase } \\
\text { Inverter }\end{array}$} & $\mathrm{P}=0$ & $\begin{array}{c}\text { Sim. } \\
\text { Result } \\
\text { Cal. } \\
\text { Result } \\
\end{array}$ & $\begin{array}{l}0.639 \\
0.637\end{array}$ & $\begin{array}{l}0.748 \\
0.728\end{array}$ & $\begin{array}{l}42.93 \\
43.14\end{array}$ & $\begin{array}{l}65.45 \\
65.23\end{array}$ & $\begin{array}{l}0.45 \\
0.45\end{array}$ & $\begin{array}{c}- \\
0.449\end{array}$ & $\begin{array}{c}- \\
1.67\end{array}$ & $\begin{array}{c}- \\
1.67\end{array}$ & $\begin{array}{c}- \\
1.67\end{array}$ \\
\hline & $\mathrm{P}=1 / 2$ & $\begin{array}{c}\text { Sim. } \\
\text { Result } \\
\text { Cal. } \\
\text { Result }\end{array}$ & $\begin{array}{l}0.628 \\
0.615\end{array}$ & $\begin{array}{l}0.738 \\
0.748\end{array}$ & $\begin{array}{l}33.6 \\
32.9\end{array}$ & $\begin{array}{l}53.02 \\
52.99\end{array}$ & $\begin{array}{l}0.442 \\
0.442\end{array}$ & $\begin{array}{c}- \\
0.464\end{array}$ & $\begin{array}{c}- \\
3.33\end{array}$ & $\begin{array}{c}- \\
1.5\end{array}$ & $\begin{array}{c}- \\
1.54\end{array}$ \\
\hline & $\mathrm{P}=1$ & $\begin{array}{c}\text { Sim. } \\
\text { Result } \\
\text { Cal. } \\
\text { Result }\end{array}$ & $\begin{array}{l}0.605 \\
0.605\end{array}$ & $\begin{array}{l}0.711 \\
0.692\end{array}$ & $\begin{array}{l}30.19 \\
29.87\end{array}$ & $\begin{array}{c}42.93 \\
42.912\end{array}$ & $\begin{array}{l}0.43 \\
0.43\end{array}$ & $\begin{array}{c}- \\
0.428\end{array}$ & $\begin{array}{c}- \\
3.33\end{array}$ & $\begin{array}{c}- \\
1.33\end{array}$ & $\begin{array}{c}- \\
1.49\end{array}$ \\
\hline \multirow{3}{*}{$\begin{array}{l}\text { Seven } \\
\text { Phase } \\
\text { Inverter }\end{array}$} & $\mathrm{P}=0$ & $\begin{array}{l}\text { Sim. } \\
\text { Result } \\
\text { Cal. } \\
\text { Result }\end{array}$ & $\begin{array}{l}0.638 \\
0.638\end{array}$ & $\begin{array}{l}0.559 \\
0.559\end{array}$ & $\begin{array}{l}45.54 \\
46.36\end{array}$ & $\begin{array}{l}92.11 \\
92.10\end{array}$ & $\begin{array}{l}0.451 \\
0.45\end{array}$ & $\begin{array}{c}- \\
0.496\end{array}$ & $\begin{array}{c}- \\
2.33\end{array}$ & $\begin{array}{c}- \\
2.33\end{array}$ & $\begin{array}{c}- \\
2.33\end{array}$ \\
\hline & $\mathrm{P}=1 / 2$ & $\begin{array}{c}\text { Sim. } \\
\text { Result } \\
\text { Cal. } \\
\text { Result } \\
\end{array}$ & $\begin{array}{l}0.632 \\
0.632\end{array}$ & $\begin{array}{l}0.552 \\
0.552\end{array}$ & $\begin{array}{c}38.42 \\
39.1\end{array}$ & $\begin{array}{l}80.55 \\
80.57\end{array}$ & $\begin{array}{l}0.447 \\
0.447\end{array}$ & $\begin{array}{c}- \\
0.48\end{array}$ & $\begin{array}{c}- \\
4.67\end{array}$ & $\begin{array}{c}- \\
1.859\end{array}$ & $\begin{array}{c}- \\
2.28\end{array}$ \\
\hline & $\mathrm{P}=1$ & $\begin{array}{c}\text { Sim. } \\
\text { Result } \\
\text { Cal. } \\
\text { Result }\end{array}$ & $\begin{array}{l}0.621 \\
0.621\end{array}$ & $\begin{array}{l}0.541 \\
0.541\end{array}$ & $\begin{array}{l}33.76 \\
33.51\end{array}$ & $\begin{array}{l}68.8 \\
68.8\end{array}$ & $\begin{array}{l}0.439 \\
0.439\end{array}$ & $\begin{array}{c}- \\
0.463\end{array}$ & $\begin{array}{c}- \\
4.67\end{array}$ & 2 & $\begin{array}{c}- \\
2.2\end{array}$ \\
\hline
\end{tabular}

Table 5. Harmonic Profile of Multiphase Inverter

\begin{tabular}{c|c|c|c|c|c|c}
\hline \multirow{4}{*}{$\mathbf{P}=\mathbf{0}$} & & $\mathbf{n = 3}$ & $\mathbf{n = 5}$ & $\mathbf{n = 7}$ & $\mathbf{n}=\mathbf{9}$ & $\mathbf{n}=\mathbf{1 1}$ \\
\cline { 2 - 7 } & $3 \mathrm{Ph}$ & 0 & 12.62 & 9.18 & 0 & 5.69 \\
& $5 \mathrm{Ph}$ & 21.2 & 0 & 9.09 & 7.07 & 5.79 \\
& $7 \mathrm{Ph}$ & 21.22 & 12.66 & 0 & 7.13 & 5.77 \\
\hline \multirow{2}{*}{$\mathbf{P = 1}$} & $3 \mathrm{Ph}$ & 0 & 10.85 & 8.03 & 0 & 4.84 \\
& $5 \mathrm{Ph}$ & 12.46 & 0 & 5.34 & 6.73 & 5.51 \\
\hline
\end{tabular}


Sangeeta Sahu, Byamakesh Nayak and Rudra Narayan Dash/

Journal of Engineering Science and Technology Review 13 (6) (2020) 130 - 136

\begin{tabular}{c|c|c|c|c|c|c|}
\hline & $7 P h$ & 16.75 & 5.78 & 0 & 2.81 \\
\hline \multirow{3}{*}{$\mathbf{P}=1 / 2$} & $3 \mathrm{Ph}$ & 0 & 3.39 & 2.27 & 0 & 5.34 \\
& $5 \mathrm{Ph}$ & 18.89 & 0 & 4.13 & 1.11 \\
& $7 \mathrm{Ph}$ & 20.02 & 10.77 & 0 & 0.91 \\
\hline
\end{tabular}

\begin{tabular}{|c|c|c|c|c|c|c|}
\hline & Sampling time & \multicolumn{3}{|l|}{$5 e-05$} \\
\hline \multirow{2}{*}{\multicolumn{3}{|c|}{$\begin{array}{l}\text { Samples per cycle } \\
\text { DC component }\end{array}$}} & \multicolumn{4}{|c|}{$=400$} \\
\hline & & & $=$ & $1.259 \mathrm{e}$ & -09 & \\
\hline Fundames & atal & & $=$ & 62.82 & peak & 42 rms) \\
\hline THD & & & $=$ & 33.608 & & \\
\hline 0 & $\mathrm{~Hz}$ & $(\mathrm{DC}):$ & & & 0.00 & $90.0^{\circ}$ \\
\hline 25 & $\mathrm{~Hz}$ & & & & 0.00 & $0.0^{\circ}$ \\
\hline 50 & $\mathrm{~Hz}$ & (Fnd): & & & 62.82 & $152.6^{\circ}$ \\
\hline 75 & $\mathrm{~Hz}$ & & & & 0.00 & $0.0^{\circ}$ \\
\hline 100 & $\mathrm{~Hz}$ & $(\mathrm{~h} 2):$ & & & 0.00 & $0.0^{\circ}$ \\
\hline 125 & $\mathrm{~Hz}$ & & & & 0.00 & $0.0^{\circ}$ \\
\hline 150 & $\mathrm{~Hz}$ & $(\mathrm{~h} 3):$ & & & 18.89 & $97.7^{\circ}$ \\
\hline 175 & $\mathrm{~Hz}$ & & & & 0.00 & $0.0^{\circ}$ \\
\hline 200 & $\mathrm{~Hz}$ & $(\mathrm{~h} 4):$ & & & 0.00 & $0.0^{\circ}$ \\
\hline 225 & $\mathrm{~Hz}$ & & & & 0.00 & $0.0^{\circ}$ \\
\hline 250 & $\mathrm{~Hz}$ & $($ h5) : & & & 0.00 & $0.0^{\circ}$ \\
\hline 275 & $\mathrm{~Hz}$ & & & & 0.00 & $0.0^{\circ}$ \\
\hline 300 & $\mathrm{~Hz}$ & $(\mathrm{~h} 6):$ & & & 0.00 & $0.0^{\circ}$ \\
\hline 325 & $\mathrm{~Hz}$ & & & & 0.00 & $0.0^{\circ}$ \\
\hline 350 & $\mathrm{~Hz}$ & $(\mathrm{~h} 7):$ & & & 4.13 & $-12.2^{\circ}$ \\
\hline 375 & $\mathrm{~Hz}$ & & & & 0.00 & $0.0^{\circ}$ \\
\hline 400 & $\mathrm{~Hz}$ & $(\mathrm{~h} 8):$ & & & 0.00 & $0.0^{\circ}$ \\
\hline 425 & $\mathrm{~Hz}$ & & & & 0.00 & $0.0^{\circ}$ \\
\hline
\end{tabular}

Fig. 4. FFT analysis of 5 phase inverter at conduction angle $\mathrm{P}=1 / 2\left(162^{0}\right)$

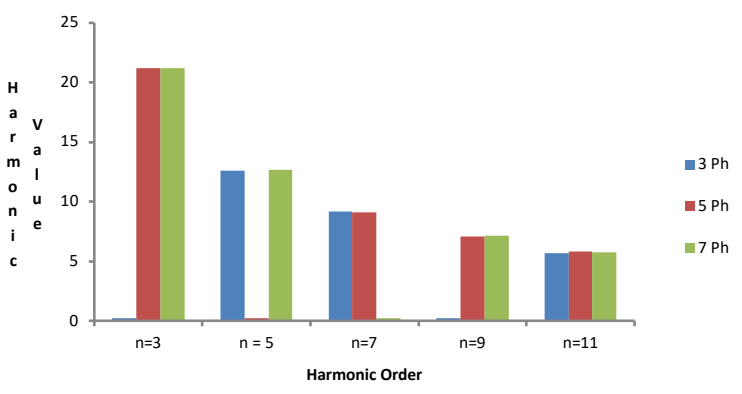

Fig. 5. Graphical representation of harmonic profile at $\mathrm{P}=0$

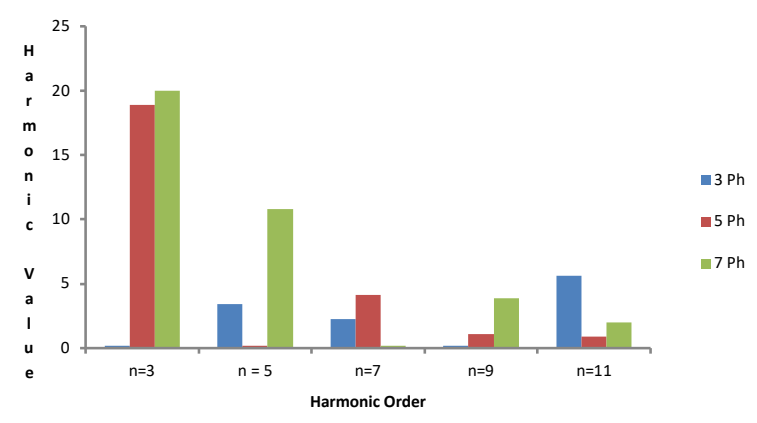

Fig. 6. Graphical representation of harmonic profile at $P=1 / 2$

Although the power handling capability of 5 phase inverter is less but the cost, switching losses and the $6^{\text {th }}$ and $12^{\text {th }}$ harmonic pulsating torque are also less compared to the 7 phase inverter which may be the right choice for hybrid electric vehicle. In addition, the conduction period of each switch for $\mathrm{P}=0,1 / 2$, and 1 are compared and found that the conduction period for $\mathrm{P}=1 / 2$ is the best choice for the above application. For $\mathrm{P}=1 / 2$ conduction angle, the level of phase voltage is more which can help to eliminate the number of dominant harmonic component using selective harmonic elimination technique. For example; if level is $\mathrm{t}$, (t-1) harmonics can be eliminated. Fig. 8 shows the power handling capacity of three phase, five phase and seven phase inverters graphically at different conduction angle $\mathrm{P}$.

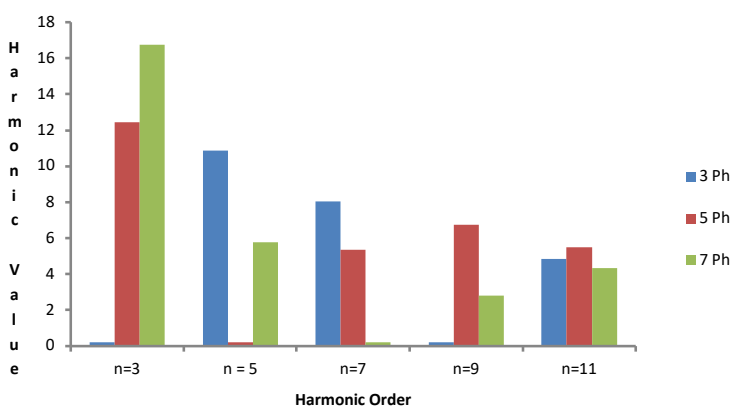

Fig. 7. Graphical representation of harmonic profile at $\mathrm{P}=1$

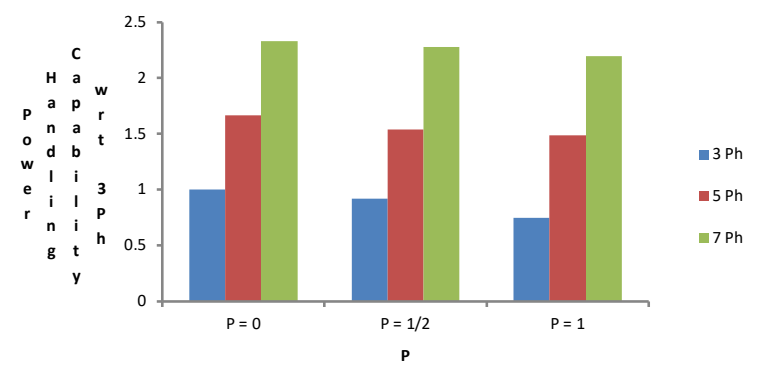

Fig. 8. Graphical representation of Power handling capacity of three phase, five phase and seven phase inverter at different $\mathrm{P}$

\section{Conclusion}

The third harmonic voltage is absent when the neutral in a star connected load is isolated. Therefore, the harmonic analysis of multiphase inverters is performed without considering the third harmonic voltage. For $\mathrm{P}=1 / 2$, the $11^{\text {th }}$ and its higher order harmonics are less in a five phase inverter as compared to three phase and seven phase inverter. Also, it can be noted that all the nth order harmonics in the five phase inverter is less than the seven phase inverter. The power handling capacity of a five phase inverter is more than the three phase inverter but less than the seven phase inverter. The fifth order harmonics are absent in five phase inverter. The switching and conduction loss increases with the increase in the phase of the inverter due to higher number of switches used. It is worth noting that the calculated values of the output voltages, harmonics and THD approximately using the derived mathematical equations match with the results found from matlab simulink.

This is an Open Access article distributed under the terms of the Creative Commons Attribution License

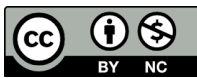




\section{References}

1. Lea Dorn-Gomba, Pierre Magne, Benjamin Danen and Ali Emadi, "On the Concept of the Multi-Source Inverter for Hybrid Electric Vehicle Powertrains," IEEE Trans. on Power Electronics, vol. 33 , no. 9 , pp. $7376-7386,2018$

2. Madhwi Kumari, Parashuram. R. Thakura and Deepak N. Badodkar, "Transient Analysis of Three-Phase High-Power Voltage Source Inverter With Nonlinearities in Hybrid Electric Vehicles," IEEE Trans. on Power Electronics, vol. 33, no. 4, pp. 3672 - 3680, 2018

3. Abbas Dehghani kiadehi, Khalil El Khamlichi Drissi and Christophe Pasquier, "Angular Modulation of Dual-Inverter Fed Open-End Motor for Electrical Vehicle Applications," IEEE Trans. on Power Electronics, vol. 31, no. 4, pp. 7376 - 7386, 2016

4. Haizhong Ye and Ali Emadi, "A Six-Phase Current Reconstruction Scheme for Dual Traction Inverters in Hybrid Electric Vehicles With a Single DC-Link Current Sensor," IEEE Trans. On Vehicular Technology, vol. 63, no. 7, pp. $3085-3093,2014$

5. P. Liu H.P. Liu, "Permanent-magnet synchronous motor drive system for electric vehicles using bidirectional Z-source inverter,' IET Electrical Systems in Transportation, vol. 2, no. 4, pp. 178-185, 2012

6. Pablo Concha Moreno-Torres, Jerome Lourd, Marcos Lafoz and Jaime R. Arribas, "Evaluation of the Magnetic Field Generated by the Inverter of an Electric Vehicle," IEEE Trans. on Magnetics, vol. 49 , no. 2 , pp. $837-844,2013$

7. S. Wei et al., "Overview on fault disposal and fault-tolerance mechanism of PMSM and its driving system," Electr. Power Automat. Equip., vol. 36, no. 10, pp. 100-107, 2016.

8. Abhishek M. Patel, "THD Comparison for 180, 120 \& 150 Degree Conduction Mode of Three Phase Inverter," in International Journal for Scientific Research \& Development, vol. 6, pp. 145 - 149, 2018

9. Jaimin Trivedi, Manori Shah, Jui Shah and Ruchit Soni, "Three Phase 150 Degree Mode of Conduction Voltage Source Inverter using Arduino," International Journal of Engineering Research \& Technology, vol. 5, pp. 272-275, 2016

10. Hamid A. Toliyat, "Analysis and Simulation of Multi-Phase Variable Speed Induction Motor Drives Under Asymmetrical Connections," in Proc. IEEE Applied Power Electronics Conf (APEC), 1996, vol. 2, pp. $586-592$

11. Bheemaiah Ch, Utkal Ranjan Muduli and Ranjan Kumar Behera, "Performance Comparison of Five-Phase Three-Level NPC to FivePhase Two-Level Voltage Source Inverter," IEEE International Conference on Power Electronics, Drives and Energy Systems, 2019.

12. M.A Inayathullaah and Dr. R. Anita, "Simulation of Five Phase Voltage Source Inverter with Different Excitation for Star Connected Load," International Journal of Engineering and Technology, vol. 6 , no. 3, 2014, pp. $1573-1580$

13. K. P. Prasad Rao, B. Krishna Veni and D. Ravithej, "Five-Leg Inverter for Five-Phase Supply," International Journal of Engineering Trends and Technology, vol. 3, pp. 144 - 152, 2012.

14. Qijun Deng, Ziyi Wang, Cheng Chen, Dariusz Czarkowski, Marian K. Kazimierczuk, Hong Zhou , and Wenshan $\mathrm{Hu}$, "Modeling and Control of Inductive Power Transfer System Supplied by Multiphase Phase-Controlled Inverter, " IEEE Trans. on Power Electronics, vol. 34, no. 9, pp. $9303-9315,2019$.

15. Chen Yong and Huang Qiu-Liang, "Four-Dimensional Space Vector PWM Strategy for Five-Phase Voltage Source Inverter," in IEEE Access, vol. 7, pp. 59013 - 59021, 2019.

16. Keng-Yuan Chen, "Space-vector pulse-width modulation for multiphase voltage source inverters considering reference order," IET Power Electronics, vol. 9, pp. 81 - 94, 2016.

17. Zicheng Liu, Zedong Zheng, Lie Xu, Kui Wang and Yongdong Li, "Current Balance Control for Symmetrical Multiphase Inverters," IEEE Trans. on Power Electronics, vol. 31, no. 6, pp. 4005 - 4012, 2016

\section{Appendix}

The mathematical equations for phase voltage and line voltage of three phase, five phase and seven phase inverters at $\mathrm{P}=0,1 / 2$ and 1 are given below. The phase voltage is derived using Fourier transform.

RMS value of phase voltage at $\mathrm{P}=0$,

$$
\begin{array}{ll}
V_{p h r m s}=\frac{4 V_{d c}}{3 \sqrt{2} n \pi}\left[1+\cos \frac{n \pi}{3}\right] & \text { 3phase } \\
V_{p h r m s}=\frac{4 V_{d c}}{5 \sqrt{2} n \pi}\left[2+\cos \frac{n \pi}{5}-\cos \frac{2 n \pi}{5}\right] & \text { 5phase } \\
V_{p h r m s}=\frac{4 V_{d c}}{7 \sqrt{2} n \pi}\left[3+\cos \frac{n \pi}{7}-\cos \frac{2 n \pi}{7}-\cos \frac{4 n \pi}{7}\right] 7 \text { phase }
\end{array}
$$

$$
V_{\text {phrms }}=\frac{4 V_{d c}}{5 \sqrt{2} n \pi}\left[\begin{array}{l}
2 \cos \frac{n \pi}{20}+ \\
+\frac{1}{2}\left(\cos \frac{3 n \pi}{20}-\cos \frac{9 n \pi}{20}+\cos \frac{5 n \pi}{20}-\cos \frac{7 n \pi}{20}\right)
\end{array}\right]
$$

RMS value of phase voltage at $\mathrm{P}=1 / 2$,

$$
\begin{aligned}
& V_{p h r m s}=\frac{4 V_{d c}}{3 \sqrt{2} n \pi}\left[\cos \frac{n \pi}{12}+\frac{1}{2}\left(\cos \frac{3 n \pi}{12}+\cos \frac{5 n \pi}{12}\right)\right] \text { 3phase } \\
& V_{p h r m s}=\frac{4 V_{d c}}{7 \sqrt{2} n \pi}\left[3 \cos \frac{n \pi}{28}+\frac{1}{2}\left(\cos \frac{3 n \pi}{28}-\cos \frac{9 n \pi}{28}-\cos \frac{17 n \pi}{28}+\cos \frac{5 n \pi}{28}-\cos \frac{7 n \pi}{28}-\cos \frac{15 n \pi}{28}\right)\right] 7 \text { phase }
\end{aligned}
$$

RMS value of phase voltage at $\mathrm{P}=1$,

$$
\begin{aligned}
& V_{p h r m s}=\frac{\sqrt{2} V_{d c}}{n \pi} \cos \frac{n \pi}{6} \\
& V_{p h r m s}=\frac{\sqrt{2} V_{d c}}{n \pi} \cos \frac{n \pi}{10}
\end{aligned}
$$$$
\text { 3phase }
$$$$
V_{p h r m s}=\frac{\sqrt{2} V_{d c}}{n \pi} \cos \frac{n \pi}{14}
$$

The RMS of fundamental value of phase voltage,

5 phase 
Sangeeta Sahu, Byamakesh Nayak and Rudra Narayan Dash/

Journal of Engineering Science and Technology Review 13 (6) (2020) 130 - 136

The RMS value of line voltage,

$V_{L 1}=2 \times V_{p h r m s 1} \times \cos 60$

$V_{L 1}=2 \times V_{p h r m s} \times \cos 54$

$V_{L 1}=2 \times V_{p h r m s 1} \times \cos 64.29$

Power is given by,

$\mathrm{P}=\frac{3 V_{p h}^{2}}{R}$

$\mathrm{P}=\frac{5 V_{p h}^{2}}{R}$

$$
\mathrm{P}=\frac{7 V_{p h}^{2}}{R}
$$

7 phase

3phase

5 phase

7 phase

The general equation of $\mathrm{n}$ phase inverter at $\mathrm{P}=0$ and 1 can be concluded from the above equations.

$$
\text { At } \mathrm{P}=0 \text {, }
$$$$
V_{p h r m s}=\frac{4 V_{d c}}{\sqrt{2} m n \pi}\left[\frac{m-1}{2}+\cos \frac{n \pi}{m}-\sum_{l=2,4,6 \ldots \frac{m-3}{2}} \cos \frac{\ln \pi}{m}\right]
$$

3phase

5 phase
AAt $\mathrm{P}=1$

$V_{p h r m s}=\frac{2 V_{d c}}{n \pi} \cos \frac{n \pi}{2 m}$

Here, $\mathrm{m}=$ number of phases and $\mathrm{n}=$ harmonic order 